two types of mask ventilation. Although the questions of this study are relevant, I believe that the design of the study was not adequate to answer these questions.

First, all patients were on NM for $>3$ months and one patient even for 70 months, whereas they trained only for 1 day on FFM. In our centre (Dept of Pulmonary Diseases/ Home mechanical ventilation, University Hospital Groningen, Groningen, The Netherlands), patients routinely need more time ( $\sim 7$ days) to adjust to their specific mask. As a result of this short time of training on FFM compared with a long-term adjustment on NM, it is not surprising that total sleep time and sleep efficiency were worse in the FFM group compared with the NM group. As a consequence of their longer experience with the NM mask, the patients also rated less leak and more comfort in the NM night compared with the FFM night. Secondly, no differences were found between pressure settings if NM was compared with FFM. However, this study will not detect any differences if pressure settings, optimalised during their review night, remained unchanged during the subsequent mask trials.

Due to the previously mentioned issues, I believe that the study by WILLSON et al. [1], with its present design, makes it difficult to draw strong conclusions. It would have been preferable to compare both mask ventilations in a parallel study of naïve patients in whom noninvasive ventilation was started. In this way, it would have been possible to compare the time it takes to adjust to either nasal mask or full face mask. If the patients were adjusted to the mask, it would be possible to determine differences in terms of sleep quality, tolerability and pressure needed to maintain an effective noninvasive ventilation.

\section{P. Wijkstra}

Dept of Pulmonary Diseases/Home mechanical ventilation, University Hospital Groningen, Groningen, The Netherlands.

\section{References}

1. Willson GN, Piper AJ, Norman M, et al. Nasal versus full face mask for noninvasive ventilation in chronic respiratory failure. Eur Respir J 2004; 23: 605-609.

DOI: $10.1183 / 09031936.04 .00054204$

\section{From the authors:}

We welcome the interest of P. Wijkstra in our paper [1]. We agree that the choice of an effective interface for noninvasive ventilation (NIV) is integral to the success of this therapy and would welcome future research in the area. We acknowledge that our study was not without methodological weaknesses, a number of which we mention in the discussion of our paper [1]. Whilst we would now consider a trial in naive patients, this was not the situation at the commencement of data collection for our study. At that time the safety and efficacy of the full face mask (FFM) for NIV during sleep was largely unknown. Our adverse experience with this interface led us to be cautious with regard to its efficacy during sleep, especially in subjects with neuromuscular disease. Indeed, this concern was borne out in the study with the observation of upper airway obstruction in one subject on FFM versus an absence of respiratory events on the nasal mask. We would still recommend careful attention to the degree of upper airway closure during sleep in subjects using a FFM.

It was with these considerations in mind that rather than choosing naïve subjects we chose a group of subjects who were receiving effective NIV therapy via a nasal mask (NM). In this sense we were well aware that the NM versus FFM comparison may be weighted toward the NM. Alternatively, the acute application of a more effective interface may be argued to have biased our study toward the FFM. The proposals of $\mathrm{P}$. Wijkstra in regard to the mask acclimatisation are well thought out and in line with many of our own clinical observations. We concur with P. Wijkstra that our study did not directly address the question of the difference in pressure requirements on the FFM. Our aim would have been more appropriately worded: To determine if the pressures titrated during NM ventilation were effective during FFM ventilation. We believe our study does show that the pressure settings titrated whilst wearing the NM will be adequate if the patient is changed to a FFM.

We would hope that the results of our study will be interpreted in light of the subjects studied and the methodology used. We would direct readers to the broader conclusions of our study that full face masks appeared to be as effective as nasal masks for the delivery of noninvasive ventilation.

\section{G.N. Willson, A.J. Piper, R.R. Grunstein}

Dept of Respiratory Medicine, Royal Prince Alfred Hospital, Camperdown, Sydney, New South Wales, Australia.

\section{References}

1. Willson GN, Piper AJ, Norman M, et al. Nasal versus full face mask for noninvasive ventilation in chronic respiratory failure. Eur Respir J 2004; 23: 605-609.

DOI: $10.1183 / 09031936.04 .00063904$

\title{
Methacholine and macrolides
}

\section{To the Editor:}

The paper by Kostadima et al. [1] provides a valuable insight into the effects of clarithromycin upon nonspecific airway hyperresponsiveness (AHR) to methacholine in asthmatics.

It is important to point out that AHR to methacholine is only very loosely related to the degree of underlying inflammation, whereas the use of an indirect bronchoconstrictor stimulus in the KOSTADIMA et al. [1] study would have provided more convincing evidence of anti-inflammatory activity [2]. Bronchoprovocation with indirect stimuli, such as adenosine monophosphate (AMP), is considered to be particularly relevant to real life situations, since cold air, exercise and allergens also act in a similar fashion, in terms of the release of inflammatory mediators from primed mast cells. Indeed, shifts in the AMP threshold are more closely related to underlying airway eosinophilic inflammation and associated with symptoms of atopic asthma than direct stimuli, such as methacholine [3, 4]. 